\title{
Do Vasodilators Interfere with Left Ventricular Remodeling in Chronic Mitral Regurgitation?
}

\author{
Roney Orismar Sampaio, Max Grinberg
}

São Paulo, SP - Brazil

The influence of vasodilators in the natural history of mitral regurgitation is controversial. If, on the one hand, they may really interfere, on the other, they may mask left ventricular myocardial dysfunction.

Three phases comprise the natural history of mitral regurgitation. In the first, increased atrial compliance and eccentric ventricular hypertrophy are observed in association with diastolic normotension. In the second phase, a transition phase, a slow increase in diastolic and left chamber pressures is observed. In the third phase, handicapping symptoms occur due to failure in compensating mechanisms and elevation in the systolic and pulmonary pressures.

It is estimated that up to $82 \%$ of the patients with mitral regurgitation due to prolapse progress to surgical indication in a 10 -year period, which is equivalent to a $20 \%$ rate of surgical indication per year ${ }^{1}$. Functional class (III or IV, New York Heart Association), advanced age, and ventricular dysfunction correlate with a greater mortality rate ${ }^{1,2}$.

In regard to the qualification of the indicators of natural history progression in patients with asymptomatic chronic mitral regurgitation, no uniformity exists. Variations in the systolic diameter between 45 and $55 \mathrm{~mm}^{2,3}$, in the diastolic diameter above $70 \mathrm{~mm}^{3}$, or correlations between cavitary increase and body surface ${ }^{4}$ are not satisfactory. However, signals of reduction in left ventricular function draw attention to the advanced character of the disease ${ }^{1-4}$.

The type of drug, its dosage, and the period of time during which it is used influence the vasodilator effect on mitral regurgitation. Greenberg et al ${ }^{5}$, using hydralazine, and Schön et $\mathrm{al}^{6}$, using quinapril, reported a reduction in left ventricular diameter and improvement of symptoms. However, this behavior has not been confirmed by other researchers ${ }^{7,8}$.

In this regard, we studied 47 patients with oligosymp-

Instituto do Coração do Hospital das Clínicas da FMUSP

Mailing address: Max Grinberg - InCor - Av. Dr. Enéas C. Aguiar, 44 - 05403-000

São Paulo, SP, Brazil - E-mail: grinberg@incor.usp.br

English version by Stela Maris C. e Gandour tomatic chronic mitral regurgitation, of whom 26 received enalapril maleate and 21 received placebo, in a randomized double-blind protocol. We observed a reduction in left ventricular and left atrial systolic and diastolic diameters ${ }^{9}$ after 12 months in the patients who received enalapril maleate as compared with those who received placebo.

How does one explain the alterations observed with the use of the vasodilator in this situation, in which an elevation of the preload and normality or reduction of the afterload exist? Hemodynamic alterations of the mitral defluxion volume, of the regurgitant volume, of the regurgitation orifice and fraction, and preservation of the systolic volume in patients who used enalapril could justify the reduction in the left cavity ${ }^{9,10}$. Left ventricular ejection fraction did not change with the use of the vasodilator, either at rest and on maximumexertion ${ }^{9}$.

Hemodynamic improvement may cause alterations in ventricular geometry and reduction in the degree of mitral valve reflux. Cardiac remodeling results from alterations in gene, molecular, cellular, and interstitial expression, manifesting as changes in cardiac dimension, shape, or function after stimulation ${ }^{11}$.

In the postinfarction model, ventricular remodeling begins in the first hours and continues for a variable period, accompanying the degree of injury ${ }^{11,12}$. Areas of dilation mixed with others of compensatory hypertrophy may be found. Therefore, global ejection fraction results from different degrees of left ventricular regional contractility.

The ventricular remodeling in mitral regurgitation depends on the degree of fluid overload and the time of evolution. If sudden, an increase in the left ventricular diastolic pressure will occur, as will immediate symptoms of pulmonary congestion. If there is time enough for adaptation, the pressure normalizes and deformation in the left ventricular cavity occurs slowly. The increase in load in mitral valve regurgitation modulates the left ventricle, favoring the maintenance of the systolic volume and absence of symptoms for a long period. The borderline between remodeling and maladaptation is difficult to identify and depends on several factors, such as time of evolution, rheumatic activity, endocar- 
dial infection, and association between valvular heart diseases. The use of a vasodilator aims at maintaining the patient in the first phase of remodeling, delaying the period characterized by maladaptation.

Angiotensin-converting enzyme inhibitors influence postinfarction prognosis, and improvement of the ejection fraction and of the systolic volume, mainly in patients with huge infarctions. The response to a drug seems to correspond directly to the size of injury, ie, the smaller the response, the smaller the ischemic necrosis ${ }^{12}$.

In regard to mitral regurgitation, the scarcity of data in the literature does not allow one to infer the same behavior. However, one should consider that the early use of a drug may positively interfere with remodeling, avoiding ventricular deformation, ie, sphericity instead of the ellipsoid form, myocardial damage, and, at last, ventricular dysfunction.

Curious alterations of the cardiopulmonary parameters on submaximal exertion, such as a reduction in oxygen consumption and pulse, and an increase in the oxygen ventilatory equivalent, were observed in our case series ${ }^{9}$. This phenomenon may be correlated with the continuous reduc- tion in the capacity for submaximal exertion, independent of the administration of vasodilators ${ }^{9}$. Therefore, functional assessment in mitral regurgitation is of paramount importance, being equivalent to anatomical assessment.

The almost exclusive use of Doppler echocardiography as a complementary method for evaluation is inadequate, because sometimes clinical and functional alterations during exercise show a greater progression of the natural history. The correspondence does not always respect the linearity with intracavitary diameters or even with the isovolumic left ventricular fraction indices.

In addition, until the present time, the use of vasodilators in chronic mitral regurgitation remains controversial ${ }^{5,6,9,10}$; it is, however, useful for the patient in an advanced functional class while waiting for surgical treatment ${ }^{5,6,9,10}$. In the asymptomatic patient, if one chooses to use vasodilators, the following measurements should guide the management: periodical and detailed clinical evaluation, determination of left ventricular function and intracavitary diameters, in addition to oxygen consumption, mainly on submaximal exertion ${ }^{9}$.

\section{References}

1. Ling LH, Enriquez-Sarano M, Seward JB, et al. Clinical outcome in mitral regurgitation due to flail leaflet. N Engl J Med 1996; 335: 1417-23.

2. Bonow RO, Carabello B, de Leon Jr AC, et al. ACC/AHA Guidelines on the management of patients with valvular heart disease: executive summary. A report of the American College of Cardiology/American Heart Association Task Force on Practice Guidelines (Committee on Management of Patients with Valvular Heart Disease). Circulation 1998; 98: 1949-84.

3. Schuler G, Peterson KL, Johnson AD, et al. Temporal response of left ventricular performance to mitral valve surgery. Circulation 1979; 59: 1218-31.

4. Zile MR, Gaasch WH, Carroll JD, Levine HJ. Chronic mitral regurgitation: predictive value of preoperative echocardiographic indexes of left ventricular function and wall stress. J Am Coll Cardiol 1984; 3: 235-42.

5. Greenberg BH, Demots H, Murphy E, Rahimtoola SH. Arterial dilators in mitral regurgitation: effects on rest and exercise hemodynamics and long term clinical follow up. Circulation 1982; 65: 181-7.
6. Schön HR, Schöter G, Barthel P, Schöming A. Quinapril therapy in patients with chronic mitral regurgitation. J Heart Valve Dis 1994; 3: 302-12.

7. Wisenbaugh T, Sinovich V, Dullabh A, Sareli P. Six month pilot study of captopril for midly symptomatic, severe isolated mitral and isolated aortic regurgitation. J Heart Valve Dis 1994; 3: 197-204.

8. Host $\mathrm{U}$, Kelbaek H, Hildebrant $\mathrm{P}$, et al. Effect of ramipril on mitral regurgitation secondary to mitral valve prolapse. Am J Cardiol 1997; 80: 655-8.

9. Sampaio RO. Influência do enalapril sobre a história natural da insuficiência mitral crônica. Estudo em pacientes oligossintomáticos. São Paulo, 2000. Tese de Doutorado. Universidade de São Paulo.

10. Gaasch WH, John RM, Aurigemma GP. Managing asymptomatic patients with chronic mitral regurgitation. Chest 1995; 108: 842-7.

11. Cohn JN, Ferrari R, Sharpe N. Cardiac remodeling - concepts and clinical implications: a consensus paper from na international forum on cardiac remodeling. J Am Coll Cardiol 2000; 35: 569-82.

12. Anversa P, Olivetti G, Capasso JM. Cellular basis of ventricular remodeling after myocardial infarction. Am J Cardiol 1991; 68: 7D-16D. 